\title{
Potencial de lixiviação de HeRbicidas Utilizados na CultuRa do Algodão em Colunas de Solo ${ }^{1}$
}

\author{
Leaching Potential of Herbicides Used in Cotton Crop under Soil Column Conditions
}

\author{
INOUE, M.H. ${ }^{2}$, SANTANA, D.C. ${ }^{3}$, OLIVEIRA JR., R.S. ${ }^{4}$, CLEMENTE, R.A. ${ }^{2}$, DALLACORT, R. ${ }^{2}$, \\ POSSAMAI, A.C.S. ${ }^{2}$, SANTANA, C.T.C. ${ }^{3}$ e PEREIRA, K.M. ${ }^{2}$
}

\begin{abstract}
RESUMO - O intenso uso de herbicidas implica a necessidade de determinar o potencial dessas substâncias em contaminar fontes aquáticas subsuperficiais. Diante dessa preocupação, o objetivo deste trabalho foi avaliar o efeito de diferentes lâminas de água sobre o potencial de lixiviação de quatro herbicidas utilizados em pré-emergência na cultura do algodão, em dois solos provenientes de Campo Novo do Parecis-MT (RQ - textura arenosa) e Tangará da Serra-MT (LV - textura argilosa). No desenvolvimento deste trabalho utilizouse a técnica de bioensaio em colunas de solo, nas quais foram simuladas irrigações de 0, 20, 40, 60, 80 e $100 \mathrm{~mm}$, após a aplicação de alachlor (RQ 2,40; LV 3,36 kg ha-1), oxyfluorfen (RQ 0,48; LV 0,72 kg ha-1), prometryne (RQ 0,75; LV 1,50 kg ha-1) e S-metolachlor (RQ 1,20; LV 1,44 $\mathrm{kg} \mathrm{ha}^{-1}$ ). Nas amostras de solo com textura arenosa (RQ), evidenciou-se que lâminas de 80 e 100 mm de água proporcionaram lixiviação até a profundidade de $10-15 \mathrm{~cm}$ do alachlor e até $15-20 \mathrm{~cm}$ do S-metolachlor. Independentemente da lâmina de água aplicada, nas amostras de RQ oxyfluorfen não ultrapassou a camada de $5-10 \mathrm{~cm}$, e o prometryne movimentou-se até a camada de $10-15 \mathrm{~cm}$ somente na lâmina de $100 \mathrm{~mm}$ de água. Nas amostras de solo com textura argilosa (LV), o oxyfluorfen não se movimentou além da camada superficial, mesmo sob as maiores lâminas de irrigação, e o prometryne atingiu $5-10 \mathrm{~cm}$ de profundidade sob lâminas de 80 e $100 \mathrm{~mm}$. Os herbicidas alachlor e S-metolachlor atingiram $10-15 \mathrm{~cm}$ de profundidade sob lâminas de 80 e $100 \mathrm{~mm}$ no LV. Evidenciou-se uma maior movimentação efetiva das moléculas de herbicidas nas amostras de solo com textura arenosa (RQ), em relação às amostras de solo com textura argilosa (LV).
\end{abstract}

Palavras-chave: águas subterrâneas, bioindicador, contaminação, impacto ambiental, textura.

\begin{abstract}
Herbicide use intensification implies in the need to determine the potential of these substances to reach groundwater. Thus, this study aimed to evaluate the influence of different irrigation depths on the leaching potential of four herbicides commonly used in preemergence cotton weed control, in samples of two soils from Campo Novo do Parecis-MT (RQ-sandy texture) and Tangará da Serra-MT (LV-clay texture). Thus, a bioassay technique in soil columns was adopted, in which water depths of 0,20,40,60,80 and $100 \mathrm{~mm}$ were simulated after application of alachlor

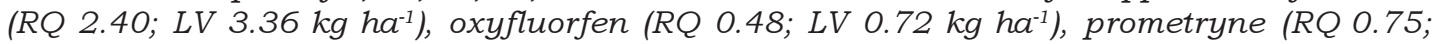
$L V 1.50 \mathrm{~kg} \mathrm{ha}^{-1}$ ) and S-metolachlor (RQ 1.20; LV $\left.1.44 \mathrm{~kg} \mathrm{ha}^{-1}\right)$. For soil samples with sandy texture (RQ), water depths of 80 and $100 \mathrm{~mm}$ led to leaching down to layers of $10-15 \mathrm{~cm}$ for alachlor and 15-20 cm for S-metolachlor.Regardless of the irrigation depth applied in the RQ samples, oxyfluorfen did not exceed the depth of 5-10 cm and prometryne could be detected at the depth of $10-15 \mathrm{~cm}$ only at water depth of $100 \mathrm{~mm}$. In columns filled with clay soil (LV), oxyfluorfen did not move beneath the surface layer, even under the highest water depths and prometryne reached the depth of $5-10 \mathrm{~cm}$ under 80 and $100 \mathrm{~mm}$. The herbicides alachlor and S-metolachlor reached $10-15 \mathrm{~cm}$ depth under water depths of 80 and $100 \mathrm{~mm}$ in the LV. A more intense downward movement of the herbicide molecules was found in sandy soil samples (RQ) than in clay texture soil samples (LV).
\end{abstract}

Keywords: groundwater, bio-indicator, contamination, environmental impact, texture.

Recebido para publicação em 15.12.2009 e na forma revisada em 12.11.2010.

2 Dep. de Agronomia, Universidade do Estado de Mato Grosso - UFMT, Tangará da Serra-MT, Brasil. Rodovia MT 358, Km 07, Caixa Postal 287, 78300-000 Tangará da Serra-MT, <miriamhinoue@hotmail.com>; ${ }^{3}$ Pós-Graduação em Agricultura, Universidade Estadual Paulista "Júlio de Mesquita Filho" - UESP, Botucatu-SP, ${ }^{4}$ Dep. de Agronomia, Universidade Estadual de Maringá, Maringá-PR. 


\section{INTRODUÇÃO}

O algodoeiro é uma planta pouco competitiva que sofre elevada interferência das plantas daninhas, as quais concorrem por água, luz, nutrientes, espaço físico e $\mathrm{CO}_{2}$. Essa cultura apresenta baixa habilidade competitiva, principalmente no crescimento inicial, que é lento, com metabolismo fotossintético $\mathrm{C}_{3}$ e raízes superficiais, fatores esses que contribuem para que a planta se torne mais vulnerável à competição e aos danos causados por cultivos mecânicos (Benedict, 1984). O nível de interferência imposto pelas plantas daninhas é um dos fatores que podem afetar essa cultura, fazendo com que ela não consiga expressar todo o seu potencial produtivo, sendo indispensável o manejo delas, principalmente, no início de seu desenvolvimento.

O cultivo do algodoeiro concentra-se, atualmente, em Mato Grosso e no oeste da Bahia. Os solos dessas regiões apresentam ampla variação de propriedades físicas e químicas. Particularmente em relação ao Mato Grosso, muitas das áreas de cultivo localizam-se em regiões que circundam as nascentes do Pantanal, o que aumenta a necessidade de estudar o comportamento de herbicidas no solo.

O controle das plantas daninhas por meio de herbicidas é utilizado em grandes áreas de plantio, por ser um método rápido e eficiente. No entanto, o uso intensivo desses produtos aumenta as preocupações com a contaminação do solo e da água, bem como com seus efeitos sobre os seres humanos e animais (Inoue et al., 2003). Além disso, os herbicidas são os agrotóxicos detectados com maior frequência fora das áreas de aplicação, aumentando o risco de contaminação ambiental (Oliveira Jr. et al., 2001).

Sabe-se que a movimentação de um herbicida no perfil do solo ocorre em todas as direções e é dependente da direção do fluxo de água. O movimento descendente dessas moléculas no perfil do solo é chamado de lixiviação. Esse evento é influenciado pelo teor e tipo de matéria orgânica, composição, tamanho e distribuição das partículas do solo, $\mathrm{pH}$ e densidade do solo, tamanho e distribuição dos poros, sendo a principal forma de transporte no solo das moléculas não voláteis e solúveis em água (Prata et al., 2003).
A lixiviação é fundamental para a incorporação superficial da maioria dos herbicidas, fazendo com que eles atinjam sementes ou plântulas em germinação, podendo, portanto, torná-lo mais ou menos eficiente no controle de plantas daninhas (Oliveira, 2001). No entanto, pesquisas revelam que a lixiviação pode também reduzir a persistência dos herbicidas, por promover o transporte desses compostos para uma região mais profunda do perfil do solo, menos explorada pelas raízes das plantas daninhas e culturas. Nesse caso, a eficácia e o potencial de injúria para as culturas em sucessão são reduzidos (Ferri \& Vidal, 2003). A lixiviação excessiva contribui também, em muitos casos, para que o herbicida atinja e contamine o lençol freático. Desse modo, entender o potencial de lixiviação dos herbicidas pode contribuir para a criação de formulações que amenizem as perdas por lixiviação desses compostos no solo (Wauchope et al., 1990; Gish et al., 1994).

O potencial de lixiviação de um herbicida pode ser avaliado por técnicas de lisímetro (Winton \& Weber, 1996), cromatografia de camada delgada de solo (Helling, 1971; Sanchez-Martin et al., 1994), entre outros métodos de avaliação que envolvem o uso de equipamentos caros e de elevado custo de manutenção. Como alternativa, podem ser utilizadas amostras deformadas em colunas de solo (Souza et al., 2000; Inoue et al., 2002; Bachega et al., 2009), com a semeadura de espécies vegetais sensiveis ao herbicida de interesse denominadas bioindicadoras.

Nesse sentido, objetivou-se no presente trabalho avaliar o potencial de lixiviação dos herbicidas alachlor, oxyfluorfen, prometryne e S-metolachlor, sob diferentes simulações de precipitações pluviais, em dois solos com texturas contrastantes provenientes de áreas cultivadas com algodão.

\section{MATERIAL E MÉTODOS}

Foram conduzidos oito ensaios em casa de vegetação, visando avaliar o potencial de lixiviação dos herbicidas alachlor, oxyfluorfen, prometryne e S-metolachlor. Para isso, foram utilizadas amostras deformadas de solo, provenientes de Campo Novo do Parecis-MT (textura arenosa) e Tangará da Serra-MT (textura 
argilosa), áreas cultivadas anteriormente com algodão há dois anos. $\mathrm{O}$ solo de textura arenosa foi classificado como Neossolo Quartzarênico (RQ), e o solo de textura argilosa, como Latossolo Vermelho (LV). As características químicas e físicas das amostras de solo estudadas são apresentadas na Tabela 1 .

As amostras foram peneiradas e devidamente acondicionadas em colunas de PVC com $30 \mathrm{~cm}$ de altura e $10 \mathrm{~cm}$ de diâmetro, anteriormente recobertas com parafina na superficie interna, visando evitar a retenção da água nas bordas internas da coluna. A borda inferior das colunas foi fechada com sombrite, para reter o solo. Após o acondicionamento do solo nas colunas, estas foram umidificadas por capilaridade durante 24 horas para saturação das amostras. Em seguida, as colunas foram mantidas sobre a bancada da casa de vegetação também por 24 horas, permitindo que o excesso de água fosse drenado.

Os herbicidas foram aplicados na fase superior das colunas, utilizando um pulverizador costal pressurizado a $\mathrm{CO}_{2}$, munido de bicos tipo leque XR110.02, mantido à pressão de trabalho de $2 \mathrm{kgf} \mathrm{cm}^{-2}$, proporcionando vazão de $200 \mathrm{~L} \mathrm{ha}^{-1}$, mantendo-se $50 \mathrm{~cm}$ entre a borda superior da coluna e a barra de aplicação. Foram ajustadas as doses dos herbicidas para cada tipo de solo. No RQ e LV, foram aplicadas, respectivamente, as seguintes doses: alachlor $\left(2,4\right.$ e $\left.3,36 \mathrm{~kg} \mathrm{ha}^{-1}\right)$, oxyfluorfen $(0,48$ e $\left.0,72 \mathrm{~kg} \mathrm{ha}^{-1}\right)$, prometryne $\left(0,75\right.$ e $\left.1,5 \mathrm{~kg} \mathrm{ha}^{-1}\right)$ e S-metolachlor $\left(1,2\right.$ e 1,44 $\left.\mathrm{kg} \mathrm{ha}^{-1}\right)$, de acordo com as recomendações de Rodrigues \& Almeida (2005).

Em seguida, foram simuladas precipitações de 0, 20, 40, 60, 80 e 100 mm, com auxílio de um equipamento simulador de chuvas. As colunas foram seccionadas longitudinalmente com uma serra elétrica, obtendo-se duas metades, que foram separadas três dias após a aplicação dos herbicidas, permanecendo assim por mais 24 horas. Após esse período, foi realizada a semeadura das espécies bioindicadoras (Brachiaria decumbens ou Cucumis sativus) longitudinalmente, sendo 25 sementes em cada metade de coluna. Utilizou-se apenas o bioindicador mais sensivel para cada herbicida; a seleção da espécie bioindicadora adequada para cada herbicida foi realizada em ensaios preliminares (dados não publicados). Vinte e um dias após a semeadura, realizaramse avaliações de fitointoxicação, nas diferentes secções da coluna $(0-5,5-10,10-15,15-20$, 20-25 e 25-30 cm de profundidade). Como critério de avaliação de fitointoxicação, adotou-se a escala de notas de 0 a $100 \%$, em que 0 corresponde a nenhuma injúria e $100 \%$ à morte das plantas.

Cada ensaio foi composto pela combinação entre um herbicida (alachlor, oxyfluorfen, prometryne ou S-metolachlor) e um solo (RQ ou LV). Foi adotado o delineamento em blocos casualizados, em esquema fatorial $6 \times 6$, com quatro repetições. Os fatores referem-se às seis faixas de profundidades na coluna $(0-5,5-10$, $10-15,15-20,20-25$ e $25-30 \mathrm{~cm})$ e às seis intensidades de irrigação $(0,20,40,60,80$ e $100 \mathrm{~mm})$. Os dados foram submetidos à análise de variância, e as médias, comparadas pelo teste de agrupamento de Scott Knott a 5\% de probabilidade.

\section{RESULTADOS E DISCUSSÃO}

Nas Tabelas 2 e 3 são apresentados os dados de fitointoxicação de $C$. sativus após a

Tabela 1 - Características químicas e físicas das amostras de solos utilizadas nos experimentos

\begin{tabular}{|c|c|c|c|c|c|c|c|c|}
\hline \multirow{2}{*}{ Solo } & \multicolumn{2}{|c|}{$\mathrm{pH}$} & $\mathrm{Al}^{3+}$ & $\mathrm{H}^{+}+\mathrm{Al}^{3+}$ & $\mathrm{Ca}^{+2}+\mathrm{Mg}^{2+}$ & $\mathrm{Ca}^{2+}$ & $\mathrm{K}^{+}$ & \multirow{2}{*}{$\frac{\mathrm{P}}{\left(\mathrm{mg} \mathrm{dm}^{-3}\right)}$} \\
\hline & $\left(\mathrm{CaCl}_{2}\right)$ & $\left(\mathrm{H}_{2} \mathrm{O}\right)$ & \multicolumn{5}{|c|}{$\left(\mathrm{cmol}_{\mathrm{c}} \mathrm{dm}^{-3}\right)$} & \\
\hline $\mathrm{RQ}^{1 /}$ & 7,10 & 7,80 & 0,00 & 1,13 & 2,75 & 2,19 & 0,02 & 46,8 \\
\hline $\mathrm{LV}^{2}$ / & 5,80 & 6,70 & 0,00 & 3,13 & 6,00 & 4,31 & 0,37 & 2,30 \\
\hline \multirow{2}{*}{ Solo } & \multicolumn{2}{|c|}{ M.O. } & CTC & \multicolumn{2}{|r|}{$\mathrm{V}$} & Areia & Silte & Argila \\
\hline & \multicolumn{2}{|c|}{$\left(\mathrm{g} \mathrm{dm}^{-3}\right)$} & $\left(\mathrm{cmol}_{\mathrm{c}} \mathrm{dm}^{-3}\right)$ & \multicolumn{2}{|r|}{$(\%)$} & \multicolumn{3}{|c|}{$\left(\mathrm{g} \mathrm{kg}^{-1}\right)$} \\
\hline $\mathrm{RQ}^{1 /}$ & \multicolumn{2}{|c|}{15,00} & 3,90 & \multicolumn{2}{|r|}{71,00} & 908,00 & 32,00 & 60,00 \\
\hline $\mathrm{LV}^{2} /$ & \multicolumn{2}{|c|}{43,00} & 9,50 & \multicolumn{2}{|r|}{67,10} & 415,00 & 147,00 & 438,00 \\
\hline
\end{tabular}

1/ RQ: Neossolo Quartzarênico (textura arenosa). ${ }^{2 /}$ LV: Latossolo Vermelho (textura argilosa). 
aplicação de alachlor em amostras de Neossolo Quartzarênico e Latossolo Vermelho, respectivamente. No solo com textura arenosa (RQ), não houve evidência de arraste vertical das moléculas de alachlor abaixo da camada superficial $(0-5 \mathrm{~cm})$ para as lâminas de 0 e $20 \mathrm{~mm}$ de água (Tabela 2). As plantas apresentaram pouca intoxicação na camada de $5-10 \mathrm{~cm}$ para a lâmina de $40 \mathrm{~mm}$, sendo maiores na mesma camada para a lâmina de $60 \mathrm{~mm}$ (Tabela 2). As evidências indicam ainda que as moléculas de alachlor atingiram a camada de $10-15 \mathrm{~cm}$ de profundidade nas lâminas de 80 e $100 \mathrm{~mm}$ (Tabela 2). Esse resultado indica a mobilidade deste herbicida em solo com textura arenosa, conforme constatado por Bowman (1990), o qual observou que o alachlor apresentou mobilidade quando aplicado em lisímetro composto de areia. Esse autor verificou que o alachlor não ultrapassou $10 \mathrm{~cm}$ de profundidade em condições de clima seco durante 21 semanas, mas, quando submetido a uma lâmina de $50 \mathrm{~mm}$, o pesticida atingiu uma profundidade de $30 \mathrm{~cm}$ e não foi detectado a partir da $12^{\mathrm{a}}$ semana. $\mathrm{O}$ alachlor pertence ao grupo químico das cloroacetamidas e é um herbicida não iônico, persistindo de 6 a 10 semanas no solo. Apresenta alto indice de sorção à matéria orgânica $\left(\mathrm{K}_{\mathrm{oc}}\right)$ de $103 \mathrm{~mL} \mathrm{~g}^{-1}$ de solo e apresenta potencial de lixiviação pelo indice de estimativa GUS (Oliveira Jr., 2007).

Nas amostras de solo com textura argilosa, não foi evidenciada mobilidade das moléculas de alachlor abaixo da camada de $0-5 \mathrm{~cm}$ com a utilização das lâminas de 0,20 e $40 \mathrm{~mm}$ (Tabela 3). Nas colunas que receberam lâminas de $60 \mathrm{~mm}$, foram detectados niveis do produto até a profundidade de $5-10 \mathrm{~cm}$. Evidenciouse ainda, em menor proporção, lixiviação até a camada de $10-15 \mathrm{~cm}$ com aplicação das lâminas de 80 e 100 mm (Tabela 3).

Nesse contexto, estudos realizados por Fawcett et al. (1994) mostraram que até 90\% de perdas das cloroacetamidas ocorrem na fase aquosa e as concentrações desses herbicidas sorvidos aos sedimentos são maiores do que aquelas dissolvidas na solução do solo. Sabese ainda que o $\mathrm{pH}$ não interfere na dissociação do produto; a degradação da molécula resulta principalmente da atividade microbiana (Rodrigues \& Almeida, 2005) e a maior sorção do alachlor ocorre em solos com teores mais elevados de argila e matéria orgânica (Ferri et al., 2006). Por outro lado, há evidências de que o aumento da temperatura exerce efeito indireto sobre a sorção das cloroacetamidas, como alachlor e metolachlor, pelo aumento da solubilidade de suas moléculas, podendo aumentar a lixiviação e diminuir a sorção dos herbicidas ao solo (Oliveira Jr. et al., 2001). Resumidamente, o herbicida alachlor apresenta grande potencial de lixiviação quando submetido a precipitações maiores que $60 \mathrm{~mm}$ logo após sua aplicação ao solo, podendo ser detectado até $10-15 \mathrm{~cm}$ de profundidade, independentemente do solo utilizado.

Nos ensaios que utilizaram amostras de $R Q$, somente foi observada pequena mobilidade das moléculas de oxyfluorfen para a camada de $5-10 \mathrm{~cm}$ com aplicação de lâminas $\geq 80 \mathrm{~mm}$ de água, evidenciando que pode ocorrer pequena movimentação das moléculas do herbicida sob precipitações intensas (Tabela 4). Por outro lado, não se detectou lixiviação das moléculas de oxyfluorfen abaixo da camada superficial $(0-5 \mathrm{~cm})$, nos ensaios realizados com amostras de LV (Tabela 5).

Tabela 2 - Fitointoxicação de pepino (C. sativus) após aplicação do herbicida alachlor $\left(2,40 \mathrm{~kg} \mathrm{ha}^{-1}\right)$ nas amostras de Neossolo Quartzarênico (textura arenosa)

\begin{tabular}{|c|c|c|c|c|c|c|}
\hline \multirow{2}{*}{$\begin{array}{l}\text { Profundidade na } \\
\text { coluna }(\mathrm{cm})\end{array}$} & \multicolumn{6}{|c|}{ Lâmina aplicada (mm) } \\
\hline & 0 & 20 & 40 & 60 & 80 & 100 \\
\hline $0-5$ & $91,3 \mathrm{Ab}$ & $90,0 \mathrm{Ab}$ & $87,5 \mathrm{Ac}$ & $91,3 \mathrm{Ab}$ & $96,3 \mathrm{Aa}$ & $90,5 \mathrm{Ac}$ \\
\hline $5-10$ & $0 \mathrm{Bd}$ & $0 \mathrm{Bd}$ & $15,0 \mathrm{Bc}$ & $93,8 \mathrm{Aa}$ & $90,0 \mathrm{Ba}$ & $91,5 \mathrm{Ab}$ \\
\hline $10-15$ & $0 \mathrm{Bb}$ & $0 \mathrm{Bb}$ & $0 \mathrm{Cb}$ & $0 \mathrm{Bb}$ & $91,5 \mathrm{Ba}$ & $87,5 \mathrm{Aa}$ \\
\hline $15-20$ & $0 \mathrm{Ba}$ & $0 \mathrm{Ba}$ & $0 \mathrm{Ca}$ & $0 \mathrm{Ba}$ & $0 \mathrm{Ca}$ & $0 \mathrm{Ba}$ \\
\hline $20-25$ & $0 \mathrm{Ba}$ & $0 \mathrm{Ba}$ & $0 \mathrm{Ca}$ & $0 \mathrm{Ba}$ & $0 \mathrm{Ca}$ & $0 \mathrm{Ba}$ \\
\hline $25-30$ & $0 \mathrm{Ba}$ & $0 \mathrm{Ba}$ & $0 \mathrm{Ca}$ & $0 \mathrm{Ba}$ & $0 \mathrm{Ca}$ & $0 \mathrm{Ba}$ \\
\hline
\end{tabular}

Médias seguidas de mesma letra, maiúscula na coluna e minúscula na linha, não diferem entre si pelo teste de agrupamento de Scott-Knott a $5 \%$ de probabilidade. $\mathrm{CV}=12,90 \%$. 
Tabela 3 - Fitointoxicação de pepino (C. sativus) após aplicação do herbicida alachlor $\left(3,36 \mathrm{~kg} \mathrm{ha}^{-1}\right)$ nas amostras de Latossolo Vermelho (textura argilosa)

\begin{tabular}{|c|c|c|c|c|c|c|}
\hline \multirow{2}{*}{$\begin{array}{c}\text { Profundidade na } \\
\text { coluna }(\mathrm{cm})\end{array}$} & \multicolumn{9}{|c|}{ Lâmina aplicada $(\mathrm{mm})$} \\
\cline { 2 - 7 } & 0 & 20 & 40 & 60 & 80 & 100 \\
\hline $0-5$ & $95,8 \mathrm{Aa}$ & $95,0 \mathrm{Aa}$ & $92,5 \mathrm{Aa}$ & $90,0 \mathrm{Aa}$ & $92,5 \mathrm{Aa}$ & $88,8 \mathrm{Aa}$ \\
\hline $5-10$ & $0 \mathrm{Bc}$ & $0 \mathrm{Bc}$ & $0 \mathrm{Bc}$ & $57,0 \mathrm{Bb}$ & $93,8 \mathrm{Aa}$ & $90,0 \mathrm{Aa}$ \\
\hline $10-15$ & $0 \mathrm{Bb}$ & $0 \mathrm{Bb}$ & $0 \mathrm{Bb}$ & $0 \mathrm{Cb}$ & $8,8 \mathrm{Ba}$ & $8,8 \mathrm{Ba}$ \\
\hline $15-20$ & $0 \mathrm{Ba}$ & $0 \mathrm{Ba}$ & $0 \mathrm{Ba}$ & $0 \mathrm{Ca}$ & $0 \mathrm{Ca}$ & $0 \mathrm{Ca}$ \\
\hline $20-25$ & $0 \mathrm{Ba}$ & $0 \mathrm{Ba}$ & $0 \mathrm{Ba}$ & $0 \mathrm{Ca}$ & $0 \mathrm{Ca}$ & $0 \mathrm{Ca}$ \\
\hline $25-30$ & $0 \mathrm{Ba}$ & $0 \mathrm{Ba}$ & $0 \mathrm{Ba}$ & $0 \mathrm{Ca}$ & $0 \mathrm{Ca}$ & $0 \mathrm{Ca}$ \\
\hline
\end{tabular}

Médias seguidas de mesma letra, maiúscula na coluna e minúscula na linha, não diferem entre si pelo teste de agrupamento de Scott-Knott a $5 \%$ de probabilidade. $\mathrm{CV}=18,64 \%$.

Tabela 4 - Fitointoxicação de $B$. decumbens após aplicação do herbicida oxyfluorfen $\left(0,48 \mathrm{~kg} \mathrm{ha}^{-1}\right)$ nas amostras de Neossolo Quartzarênico (textura arenosa)

\begin{tabular}{|c|c|c|c|c|c|c|}
\hline \multirow{2}{*}{$\begin{array}{c}\text { Profundidade na } \\
\text { coluna (cm) }\end{array}$} & \multicolumn{9}{|c|}{ Lâmina aplicada (mm) } & 60 & \multicolumn{2}{c|}{80} & 100 \\
\cline { 2 - 7 } & 0 & 20 & 40 & $91,3 \mathrm{Aa}$ & $88,8 \mathrm{Aa}$ & $92,5 \mathrm{Aa}$ \\
\hline $0-5$ & $90,0 \mathrm{Aa}$ & $95,0 \mathrm{Aa}$ & $88,8 \mathrm{Aa}$ & $0 \mathrm{Bc}$ & $20,0 \mathrm{Bb}$ & $43,8 \mathrm{Ba}$ \\
\hline $5-10$ & $0 \mathrm{Bc}$ & $0 \mathrm{Bc}$ & $0 \mathrm{Bc}$ & $0 \mathrm{Ba}$ & $0 \mathrm{Ca}$ & $0 \mathrm{Ca}$ \\
\hline $10-15$ & $0 \mathrm{Ba}$ & $0 \mathrm{Ba}$ & $0 \mathrm{Ba}$ & $0 \mathrm{Ca}$ & $0 \mathrm{Ca}$ \\
\hline $15-20$ & $0 \mathrm{Ba}$ & $0 \mathrm{Ba}$ & $0 \mathrm{Ba}$ & $0 \mathrm{Ba}$ & $0 \mathrm{Ca}$ & $0 \mathrm{Ca}$ \\
\hline $20-25$ & $0 \mathrm{Ba}$ & $0 \mathrm{Ba}$ & $0 \mathrm{Ba}$ & $0 \mathrm{Ba}$ & $0 \mathrm{Ca}$ & $0 \mathrm{Ca}$ \\
\hline $25-30$ & $0 \mathrm{Ba}$ & $0 \mathrm{Ba}$ & $0 \mathrm{Ba}$ & $0 \mathrm{Ba}$ & $0 \mathrm{Ca}$ \\
\hline
\end{tabular}

Médias seguidas de mesma letra, maiúscula na coluna e minúscula na linha, não diferem entre si pelo teste de agrupamento de Scott-Knott a $5 \%$ de probabilidade. $\mathrm{CV}=21,92 \%$.

Tabela 5 - Fitointoxicação de $B$. decumbens após aplicação do herbicida oxyfluorfen $\left(0,72 \mathrm{~kg} \mathrm{ha}^{-1}\right)$ nas amostras de Latossolo Vermelho (textura argilosa)

\begin{tabular}{|c|c|c|c|c|c|c|}
\hline \multirow{2}{*}{$\begin{array}{c}\text { Profundidade na } \\
\text { coluna }(\mathrm{cm})\end{array}$} & 0 & 20 & 40 & 60 & \multicolumn{7}{c|}{ Lâmina aplicada (mm) } & 100 \\
\hline & $0,0 \mathrm{Aa}$ & $90,0 \mathrm{Aa}$ & $90,0 \mathrm{Aa}$ & $88,8 \mathrm{Aa}$ & $87,5 \mathrm{Aa}$ & $86,3 \mathrm{Aa}$ \\
\hline $0-5$ & $0 \mathrm{Ba}$ & $0 \mathrm{Ba}$ & $0 \mathrm{Ba}$ & $0 \mathrm{Ba}$ & $0 \mathrm{Ba}$ & $0 \mathrm{Ba}$ \\
\hline $5-10$ & $0 \mathrm{Ba}$ & $0 \mathrm{Ba}$ & $0 \mathrm{Ba}$ & $0 \mathrm{Ba}$ & $0 \mathrm{Ba}$ & $0 \mathrm{Ba}$ \\
\hline $10-15$ & $0 \mathrm{Ba}$ & $0 \mathrm{Ba}$ & $0 \mathrm{Ba}$ & $0 \mathrm{Ba}$ & $0 \mathrm{Ba}$ & $0 \mathrm{Ba}$ \\
\hline $15-20$ & $0 \mathrm{Ba}$ & $0 \mathrm{Ba}$ & $0 \mathrm{Ba}$ & $0 \mathrm{Ba}$ & $0 \mathrm{Ba}$ & $0 \mathrm{Ba}$ \\
\hline $20-25$ & $0 \mathrm{Ba}$ & $0 \mathrm{Ba}$ & $0 \mathrm{Ba}$ & $0 \mathrm{Ba}$ & $0 \mathrm{Ba}$ & $0 \mathrm{Ba}$ \\
\hline $25-30$ & 90 &
\end{tabular}

Médias seguidas de mesma letra, maiúscula na coluna e minúscula na linha, não diferem entre si pelo teste de agrupamento de Scott-Knott a $5 \%$ de probabilidade. $\mathrm{CV}=17,43 \%$.

Esses resultados estão de acordo com a literatura, segundo a qual o herbicida oxyfluorfen é praticamente imóvel no solo (Rodrigues \& Almeida, 2005), apresentando índice de adsorção $\left(\mathrm{K}_{\mathrm{oc}}\right)$ de $100.000 \mathrm{~mL} \mathrm{~g}^{-1}$ de solo e persistência média de 30 dias. Esses autores afirmam ainda que a adsorção de oxyfluorfen é proporcional ao teor de matéria orgânica. Em trabalho realizado por Peñaherrera-Colina et al. (2005), em que se testou a persistência e a mobilidade de 
oxyfluorfen em solo, foram obtidos resultados não significativos devido à pouca mobilidade do herbicida no solo em relação à massa seca total da planta bioindicadora colhida no 10으 dia a $20 \mathrm{~cm}$ de profundidade e no 40 dia após a aplicação nas profundidades de 5-10 e 10-15 cm. Do mesmo modo, Yen et al. (2003) concluíram que a possibilidade de contaminação de águas subterrâneas por oxyfluorfen é muito baixa, podendo ocorrer apenas quando o solo apresenta niveis muito baixos de matéria orgânica. Assim, a baixa concentração das moléculas de oxyfluorfen abaixo da camada superficial $(0-5 \mathrm{~cm})$ nas amostras de RQ após a ocorrência de intensas precipitações pode ser atribuída ao baixo teor de matéria orgânica deste $\left(15 \mathrm{~g} \mathrm{dm}^{-3}\right)$, quando comparada com as amostras de LV (43 $\mathrm{g} \mathrm{dm}^{-3}$ ) (Tabela 1).

O herbicida prometryne nas amostras de solo RQ não apresentou mobilidade abaixo da camada de $0-5 \mathrm{~cm}$ para lâminas de até $40 \mathrm{~mm}$ de água (Tabela 6). No entanto, verificou-se que lâminas de 60 e $80 \mathrm{~mm}$ proporcionaram sintomas de intoxicação nas plantas bioindicadoras até a profundidade de $5-10 \mathrm{~cm}$. Com a lâmina de $100 \mathrm{~mm}$, o herbicida foi lixiviado até a camada de $10-15 \mathrm{~cm}$ (Tabela 6). Nesse sentido, a estimativa do potencial de lixiviação calculada pelo índice GUS classifica a lixiviação da molécula como intermediária (Inoue et al., 2003).

Os resultados com solo de textura argilosa (LV), apresentados na Tabela 7, indicam que houve movimentação das moléculas de prometryne até a profundidade de $5-10 \mathrm{~cm}$ somente nas colunas que receberam lâminas de 80 e $100 \mathrm{~mm}$ de água. Portanto, ao comparar os solos, o maior potencial de lixiviação observado nas amostras de RQ pode ser atribuído ao menor teor de argila ( $\left.60 \mathrm{~g} \mathrm{~kg}^{-1}\right)$ e carbono orgânico $\left(25,86 \mathrm{~g} \mathrm{dm}^{-3}\right)$ presente neste solo, em relação ao LV (438 $\mathrm{g} \mathrm{kg}^{-1}$ de argila e $74,13 \mathrm{~g} \mathrm{dm}^{-3}$ de carbono orgânico).

O prometryne é um herbicida de dissociação básica, para o qual a retenção das moléculas é influenciada principalmente pelo teor de carbono e argila do solo, e que apresenta índice de sorção $\left(K_{o c}\right)$ entre 400 e $500 \mathrm{~mL} \mathrm{~g}^{-1}$ de solo (Oliveira Jr., 2007). Esse fator torna o prometryne sensivel às propriedades do solo, como observado em trabalho realizado por Cao et al. (2008), que estudaram a mobilidade do prometryne em dois solos diferentes e verificaram que ele teve moderada a baixa mobilidade, a qual foi afetada pela propriedade de cada solo especificamente. Ainda, estudos de Prata et al. (2001) evidenciaram que herbicidas do grupo das triazinas, como o prometryne, apresentam de baixa a média solubilidade em água e reduzida velocidade de degradação, fatores que podem aumentar a persistência da molécula no solo e a probabilidade de contaminação de lençóis freáticos.

Independentemente da lâmina de água aplicada, as moléculas de S-metolachlor foram carreadas para profundidades superiores a 0-5 cm nas amostras de RQ. Com as lâminas de 20 a $60 \mathrm{~mm}$ de água, notaram-se sintomas de intoxicação nas plantas de $B$. decumbens até a camada de $10-15 \mathrm{~cm}$ de profundidade (Tabela 8). Contudo, verificou-se ainda que lâminas iguais ou maiores que $80 \mathrm{~mm}$ proporcionaram movimentação do herbicida até a profundidade de $15-20 \mathrm{~cm}$ (Tabela 8 ). De modo geral, quanto maior a lâmina de precipitação, maior foi o arraste vertical das moléculas de S-metolachlor, o que implica elevação do potencial de contaminação de águas subterrâneas. Em estudos com o herbicida S-metolachlor, Burgard et al. (1993) e Procópio et al. (2001) concluíram que a profundidade de lixiviação das moléculas desse herbicida depende da ocorrência e da intensidade de chuvas ou irrigação que ocorrem após a aplicação, além dos teores de argila e de matéria orgânica no solo. No trabalho realizado por Bowman (1988), verificou-se que o S-metolachlor aplicado sob uma lâmina de água de $50,8 \mathrm{~mm}$ permitiu o transporte do herbicida até $40 \mathrm{~cm}$ de profundidade, sugerindo, nesse mesmo estudo, que S-metolachlor, aplicado em solos agrícolas nas doses recomendadas, seria minimamente lixiviado sob condições meteorológicas normais, a menos que uma chuva forte ocorresse logo após a aplicação. Nesse caso, o índice GUS classifica o S-metolachlor como de alto potencial de lixiviação, uma vez que a sorção é intermediária $\left(\mathrm{K}_{\mathrm{oc}}=200 \mathrm{~mL} \mathrm{~g}^{-1}\right.$ de solo) e apresenta alta solubilidade em água (480 $\mathrm{mg} \mathrm{L}^{-1}$ a $25{ }^{\circ} \mathrm{C}$ ) (Oliveira Jr., 2007).

Os resultados da análise de fitointoxicação do bioindicador no solo com textura argilosa (LV) não evidenciaram a presença do herbicida 
além da camada de $5-10 \mathrm{~cm}$ para as lâminas de até $60 \mathrm{~mm}$ de água. Para as lâminas de 80 e $100 \mathrm{~mm}$, as moléculas foram detectadas até a profundidade de $10-15 \mathrm{~cm}$, porém com sintomas relativamente bem menores, quando comparadas às amostras de solo com textura arenosa (Tabelas 8 e 9). Segundo Oliveira Jr. (2007), os herbicidas não iônicos, como o S-metolachlor, têm a degradação e dissociação relacionada ao teor de matéria orgânica e argila do solo. Solos orgânicos têm menor lixiviação de S-metolachlor e, além disso, esta

Tabela 6 - Fitointoxicação de pepino (C. sativus) após aplicação do herbicida prometryne $\left(0,75 \mathrm{~kg} \mathrm{ha}^{-1}\right)$ nas amostras de Neossolo Quartzarênico (textura arenosa)

\begin{tabular}{|c|c|c|c|c|c|c|}
\hline \multirow{2}{*}{$\begin{array}{l}\text { Profundidade na } \\
\text { coluna }(\mathrm{cm})\end{array}$} & \multicolumn{6}{|c|}{ Lâmina aplicada (mm) } \\
\hline & 0 & 20 & 40 & 60 & 80 & 100 \\
\hline $0-5$ & $83,8 \mathrm{Aa}$ & 85,0 Aa & $82,0 \mathrm{Aa}$ & $75,0 \mathrm{Ab}$ & $77,0 \mathrm{Ac}$ & $62,3 \mathrm{Ad}$ \\
\hline $5-10$ & $0 \mathrm{Bb}$ & $0 \mathrm{Bb}$ & $0 \mathrm{Bb}$ & $50,8 \mathrm{Ba}$ & $46,3 \mathrm{Ba}$ & $63,3 \mathrm{Aa}$ \\
\hline $10-15$ & $0 \mathrm{Bb}$ & $0 \mathrm{Bb}$ & $0 \mathrm{Bb}$ & $0 \mathrm{Cb}$ & $0 \mathrm{Cb}$ & $22,5 \mathrm{Ba}$ \\
\hline $15-20$ & $0 \mathrm{Ba}$ & $0 \mathrm{Ba}$ & $0 \mathrm{Ba}$ & $0 \mathrm{Ca}$ & $0 \mathrm{Ca}$ & $0 \mathrm{Ca}$ \\
\hline $20-25$ & $0 \mathrm{Ba}$ & $0 \mathrm{Ba}$ & $0 \mathrm{Ba}$ & $0 \mathrm{Ca}$ & $0 \mathrm{Ca}$ & $0 \mathrm{Ca}$ \\
\hline $25-30$ & $0 \mathrm{Ba}$ & $0 \mathrm{Ba}$ & $0 \mathrm{Ba}$ & $0 \mathrm{Ca}$ & $0 \mathrm{Ca}$ & $0 \mathrm{Ca}$ \\
\hline
\end{tabular}

Médias seguidas de mesma letra, maiúscula na coluna e minúscula na linha, não diferem entre si pelo teste de agrupamento de Scott-Knott a $5 \%$ de probabilidade. $\mathrm{CV}=26,48 \%$.

Tabela 7 - Fitointoxicação de pepino (C. sativus) após aplicação do herbicida prometryne (1,50 $\left.\mathrm{kg} \mathrm{ha}^{-1}\right)$ nas amostras de Latossolo Vermelho (textura argilosa)

\begin{tabular}{|c|c|c|c|c|c|c|}
\hline \multirow{2}{*}{$\begin{array}{l}\text { Profundidade na } \\
\text { coluna }(\mathrm{cm})\end{array}$} & \multicolumn{6}{|c|}{ Lâmina aplicada (mm) } \\
\hline & 0 & 20 & 40 & 60 & 80 & 100 \\
\hline $0-5$ & $82,5 \mathrm{Aa}$ & $85,0 \mathrm{Aa}$ & $83,8 \mathrm{Aa}$ & 82,0 Aa & $79,3 \mathrm{Aa}$ & 83,8 Aa \\
\hline $5-10$ & $0 \mathrm{Bc}$ & $0 \mathrm{Bc}$ & $0 \mathrm{Bc}$ & $0 \mathrm{Bc}$ & $5,0 \mathrm{Bb}$ & $12,5 \mathrm{Ba}$ \\
\hline $10-15$ & $0 \mathrm{Ba}$ & $0 \mathrm{Ba}$ & $0 \mathrm{Ba}$ & $0 \mathrm{Ba}$ & $0 \mathrm{Ca}$ & $0 \mathrm{Ca}$ \\
\hline $15-20$ & $0 \mathrm{Ba}$ & $0 \mathrm{Ba}$ & $0 \mathrm{Ba}$ & $0 \mathrm{Ba}$ & $0 \mathrm{Ca}$ & $0 \mathrm{Ca}$ \\
\hline $20-25$ & $0 \mathrm{Ba}$ & $0 \mathrm{Ba}$ & $0 \mathrm{Ba}$ & $0 \mathrm{Ba}$ & $0 \mathrm{Ca}$ & $0 \mathrm{Ca}$ \\
\hline $25-30$ & $0 \mathrm{Ba}$ & $0 \mathrm{Ba}$ & $0 \mathrm{Ba}$ & $0 \mathrm{Ba}$ & $0 \mathrm{Ca}$ & $0 \mathrm{Ca}$ \\
\hline
\end{tabular}

Médias seguidas de mesma letra, maiúscula na coluna e minúscula na linha, não diferem entre si pelo teste de agrupamento de Scott-Knott a $5 \%$ de probabilidade. $\mathrm{CV}=18,18 \%$.

Tabela 8 - Fitointoxicação de B. decumbens após aplicação do herbicida S-metolachlor $\left(1,20 \mathrm{~kg} \mathrm{ha}^{-1}\right)$ nas amostras de Neossolo Quartzarênico (textura arenosa)

\begin{tabular}{|c|c|c|c|c|c|c|}
\hline \multirow{2}{*}{$\begin{array}{l}\text { Profundidade na } \\
\text { coluna }(\mathrm{cm})\end{array}$} & \multicolumn{6}{|c|}{ Lâmina aplicada (mm) } \\
\hline & 0 & 20 & 40 & 60 & 80 & 100 \\
\hline $0-5$ & $92,5 \mathrm{Ab}$ & $96,3 \mathrm{Aa}$ & $100,0 \mathrm{Aa}$ & $91,3 \mathrm{Ab}$ & $82,5 \mathrm{Ac}$ & $83,8 \mathrm{Ac}$ \\
\hline $5-10$ & $0 \mathrm{Bf}$ & $33,8 \mathrm{Be}$ & $92,5 \mathrm{Ba}$ & $85,0 \mathrm{Bb}$ & $50,0 \mathrm{Bd}$ & $63,8 \mathrm{Bc}$ \\
\hline $10-15$ & $0 \mathrm{Bb}$ & $5,0 \mathrm{Cb}$ & $0 \mathrm{Cb}$ & $0 \mathrm{Cb}$ & $15,0 \mathrm{Ca}$ & $20,0 \mathrm{Ca}$ \\
\hline $15-20$ & $0 \mathrm{Ba}$ & $0 \mathrm{Ca}$ & $0 \mathrm{Ca}$ & $0 \mathrm{Ca}$ & $0 \mathrm{Da}$ & $0 \mathrm{Da}$ \\
\hline $20-25$ & $0 \mathrm{Ba}$ & $0 \mathrm{Ca}$ & $0 \mathrm{Ca}$ & $0 \mathrm{Ca}$ & $0 \mathrm{Da}$ & $0 \mathrm{Da}$ \\
\hline $25-30$ & $0 \mathrm{Ba}$ & $0 \mathrm{Ca}$ & $0 \mathrm{Ca}$ & $0 \mathrm{Ca}$ & $0 \mathrm{Da}$ & $0 \mathrm{Da}$ \\
\hline
\end{tabular}

Médias seguidas de mesma letra, maiúscula na coluna e minúscula na linha, não diferem entre si pelo teste de agrupamento de Scott-Knott a $5 \%$ de probabilidade. $\mathrm{CV}=13,59 \%$. 
Tabela 9 - Fitointoxicação de B. decumbens após aplicação do herbicida S-metolachlor $\left(1,44 \mathrm{~kg} \mathrm{ha}^{-1}\right)$ nas amostras de Latossolo Vermelho (textura argilosa)

\begin{tabular}{|c|c|c|c|c|c|c|}
\hline \multirow{2}{*}{$\begin{array}{c}\text { Profundidade na } \\
\text { coluna (cm) }\end{array}$} & \multicolumn{6}{|c|}{ Lâmina aplicada (mm) } \\
\cline { 2 - 7 } & 0 & 20 & 40 & 60 & 80 & 100 \\
\hline $0-5$ & $92,5 \mathrm{Ab}$ & $96,3 \mathrm{Aa}$ & $100,0 \mathrm{Aa}$ & $91,3 \mathrm{Ab}$ & $82,5 \mathrm{Ac}$ & $83,8 \mathrm{Ac}$ \\
\hline $5-10$ & $0 \mathrm{Bf}$ & $33,8 \mathrm{Be}$ & $92,5 \mathrm{Ba}$ & $85,0 \mathrm{Bb}$ & $50,0 \mathrm{Bd}$ & $63,8 \mathrm{Bc}$ \\
\hline $10-15$ & $0 \mathrm{Bb}$ & $5,0 \mathrm{Cb}$ & $0 \mathrm{Cb}$ & $0 \mathrm{Cb}$ & $15,0 \mathrm{Ca}$ & $20,0 \mathrm{Ca}$ \\
\hline $15-20$ & $0 \mathrm{Ba}$ & $0 \mathrm{Ca}$ & $0 \mathrm{Ca}$ & $0 \mathrm{Ca}$ & $0 \mathrm{Da}$ & $0 \mathrm{Da}$ \\
\hline $20-25$ & $0 \mathrm{Ba}$ & $0 \mathrm{Ca}$ & $0 \mathrm{Ca}$ & $0 \mathrm{Ca}$ & $0 \mathrm{Da}$ & $0 \mathrm{Da}$ \\
\hline $25-30$ & $0 \mathrm{Ba}$ & $0 \mathrm{Ca}$ & $0 \mathrm{Ca}$ & $0 \mathrm{Ca}$ & $0 \mathrm{Da}$ & $0 \mathrm{Da}$ \\
\hline
\end{tabular}

Médias seguidas de mesma letra, maiúscula na coluna e minúscula na linha, não diferem entre si pelo teste de agrupamento de Scott-Knott a $5 \%$ de probabilidade. $\mathrm{CV}=15,62 \%$.

é inibida em solos com alto teor de argila e/ ou silte (Extoxnet, 2000). Como pode ser observado por Singh (2003), esterco de vaca e ureia aumentaram a sorção de S-metolachlor no solo, e essas alterações reduziram as perdas por lixiviação do herbicida, mostrando que os solos orgânicos são eficazes em restringir o movimento de S-metolachlor. Assim, sob precipitações semelhantes, em solos de textura mais leve, as moléculas desse herbicida são carreadas para profundidades maiores do que em solos de textura argilosa. Nesse contexto, S-metolachlor possui potencial de contaminação de águas subterrâneas, visto que, entre os pesticidas detectados por Pittman $\&$ Berndt (2003) em águas subterrâneas provenientes de áreas agrícolas do sul do estado da Geórgia (EUA), ele foi detectado em $67 \%$ das amostras analisadas.

Conclui-se que lâminas de água superiores a $60 \mathrm{~mm}$ proporcionaram maior movimentação efetiva das moléculas de herbicidas nas amostras de solo com textura arenosa (RQ), em relação às de textura argilosa (LV). Na comparação das médias, entre os herbicidas testados, S-metolachlor foi o que mostrou maior potencial de lixiviação, especialmente em solo de textura arenosa.

\section{LITERATURA CITADA}

BACHEGA, T. F. et al. Lixiviação de sulfentrazone e amicarbazone em colunas de solo com adição de óleo mineral. Planta Daninha, v. 27, n. 2, p. 363-370, 2009.

BENEDICT, C. R. Physiology. In: KOEHL, R. J.; LEWIS, C. F. Cotton. Madison: American Society of Agronomy, 1984. p. 151-201 (Series Agronomy, 24).
BOWMAN, B. T. Mobility and persistence of alachlor, atrazine and metolachlor in plainfield sand, and atrazine and isazofos in honeywood silt loam, using field lysimeters.

Environ. Toxic. Chem., v. 9, p. 453-461, 1990.

BOWMAN, B. T. Mobility and persistence of metolachlor and aldicarb in field lysimeters. J. Environ. Qual., v. 17, n. 4, p. 689-694, 1988.

BURGARD, D. J. et al. Metolachlor distribution in a sandy soil under irrigated potato production. Weed Sci., v. 41, n. 4, p. 648-655, 1993.

$\mathrm{CAO}$, J. et al. Effects of SOM, surfactant and $\mathrm{pH}$ on the sorption-desorption and mobility of prometryne in soils Chemosphere, v. 70, p. 2127-2134, 2008

EXTOXNET: Extension Toxicology Network. Metolachlor (Dual) herbicide profile 2/85. Sacramento: Department of Pesticide Regulation, 2000

FAWCETT, R. S. et al. The impact of conservation tillage on pesticide runoff into surface water: a review and analysis. J. Soil Water Conser., v. 49, n. 1, p. 126-135, 1994

FERRI, M. V. W. et al. Sorção do herbicida alachlor em solos com diferentes conteúdos de argila e carbono orgânico. 2006. Disponível em: <http://www.ufsm.br/ppgcs/ congressos/LINKS\%20DO\%20CBCS\%202003/ CBCS2003\%20EM\%20PDF/30.pdf>. Acesso em: 13 jun. 2009.

FERRI, M. V. W.; VIDAL, R. A. Persistência do herbicida acetochlor em função de sistemas de preparo e cobertura com palha. Ci. Rural, v. 33, n. 3, p. 399-404, 2003.

GISH, T. G.; SHIRMOHAMMADI, A.; WIENHOLD, B. J. Field-scale mobility and persistence of commercial and starchencapsulated atrazine and alachlor. J. Environ. Qual., v. 23, p. $355-359,1994$. 
HELLING, C. S. Pesticide mobility in soils. III. Influence of soil properties. Soil Sci. Soc. Am. Proc., v. 35, p. 743-748, 1971.

INOUE, M. H. et al. Calagem e o potencial de lixiviação de imazaquin em colunas de solo. Planta Daninha, v. 20, n. 1, p. 125-132, 2002.

INOUE, M. H. et al. Critérios para avaliação do potencial de lixiviação dos herbicidas comercializados no Estado do Paraná Planta Daninha, v. 21, n. 2, p. 313-323, 2003.

OLIVEIRA, M. F. Comportamento de herbicidas no ambiente. In: OLIVEIRA JR., R. S.; CONSTANTIN, J. Plantas daninhas e seu manejo. Guaíba: Agropecuária, 2001. p. 315-362.

OLIVEIRA JR., R. S.; KOSKINEN, W. C.; FERREIRA, F. A. Sorption and leaching potential of herbicides on Brazilian soils. Weed Res., v. 41, p. 97-111, 2001.

OLIVEIRA JR., R. S. Comportamento dos herbicidas residuais no solo: relação entre parâmetros físico-químicos e atributos do solo. Maringá: EDUEM, 2007.

PEÑAHERRERA-COLINA, L. A. et al. Persistência biológica de ametryn, diuron e oxyfluorfen no solo. Ci. Agrotec., v. 29, n. 5, p. 980-987, 2005.

PITTMAN, J. R.; BERNDT, M. P. Occurrence of herbicide degradation compounds in streams and ground water in agricultural areas of southern Georgia, 2002. In: HATCHER, K. J. GEORGIA WATER RESOURCES CONFERENCE, 2003, Athens. Anais... Athens: Institute of Ecology, The University of Georgia, 2003. v. 2. p. 856-859, 2003.

PRATA, F. et al. Degradação e sorção de ametrina em dois solos com aplicação de vinhaça. Pesq. Agropec. Bras., v. 36, n. 7, p. $975-981,2001$.
PRATA, F. et al. Glyphosate sorption and desorption in soils with different phosphorous levels. Sci. Agric., v. 60, n. 1, p. $175-180,2003$.

PROCÓPIO, S. O. et al. Efeito da irrigação inicial na profundidade de lixiviação do herbicida S-metolachlor em diferentes tipos de solos. Planta Daninha, v. 19, n. 3, p. 409-417, 2001.

RODRIGUES, B. N.; ALMEIDA, F. S. Guia de herbicidas. 5.ed. Londrina: Autores, 2005.

SANCHEZ-MARTIN, M. J.; CRISANTO, T.; ARIENZO, $\mathrm{M}$. Evaluation of the mobility of $\mathrm{C}^{14}$ - labelled pesticides in soils by thin layer chromatography using a linear analyser. J. Environ. Sci . Health, Part B, v. 3, p. 473-484, 1994.

SINGH, N. Organic manure and urea effect on metolachlor transport through packed soil columns. J. Environ. Qual., v. 32, p. 1743-1749, 2003.

SOUZA, A. P. et al. Lixiviação de glyphosate e imazapyr em solos com diferentes texturas e composição química. I. Método do bioensaio. Planta Daninha, v. 18, n. 1, p. 5-16, 2000 .

WAUCHOPE, R. D.; WILLIAMS, R. G.; MARTI, L. R. Runoff of sulfometurom-methil and cyanazine from small plots: effects of formulation and grass cover. J. Environ. Qual., v. 19, n. 1, p. 119-125, 1990.

WINTON, K.; WEBER, J. B. A review of field lysimeter studies to describe the environmental fate of pesticides. Weed Technol., v. 10, p. 202-209, 1996.

YEN, J. H.; SHEU, W. S.; WANG, Y. S. Dissipation of the herbicide oxyfluorfen in subtropical soils and its potential to contaminate groundwater. Ecotoxicol. Environ. Safety, v. 54, n. 1, p. 151-156, 2003. 